\title{
Evaluation of Brainstem Involvement in Multiple Sclerosis
}

\author{
Magdalena Krbot Skorić, Ivan Adamec, Vesna Nesek Mađarić, Mario Habek
}

\begin{abstract}
Background/Aims: The aim of the present study was to determine the optimum method to detect brainstem lesions in patients with Multiple Sclerosis (MS). Methods: 72 patients with the diagnosis of relapsing-remitting MS were prospectively included. Brainstem functional system score (BSFS) (part of the expanded disability status scale (EDSS) evaluating brainstem symptomatology) was calculated. Magnetic resonance imaging (MRI) was performed on 1.5T and T1, T2, PD and fluid-attenuated inversion recovery (FLAIR) sequences were analyzed for presence of brainstem lesions. Auditory evoked potentials (AEP) and ocular and cervical vestibular evoked myogenic potentials (oVEMP and cVEMP) were performed according to the standardized protocol. Results: From 72 patients, $18(25 \%)$ had clinical involvement of the brainstem. MRI showed brainstem involvement in 29 (40\%) patients. Of the neurophysiological tests, AEP showed pathological result in $16(22 \%)$ patients, oVEMP in 36 (50\%) patients, cVEMP in 18 (25\%) patients, and VEMP (combination of oVEMP and cVEMP) in $45(63 \%)$ patients. VEMP detected brainstem lesions in higher percentage than clinical examination, MRI and AEP, which was statistically significant $(<0.0001,0.012$ and $<0.0001$, respectively). Conclusions: Results of the present study have shown that VEMPs are the optimal method to detect brainstem lesions in multiple sclerosis and that they detect them significantly better than clinical examination, AEP or MRI.
\end{abstract}

RÉSUMÉ: Évaluation de l'atteinte du tronc cérébral dans la sclérose en plaques. Contexte/Objectifs : Le but de cette étude était de déterminer quel est le meilleur moyen de détection des lésions du tronc cérébral chez les patients atteints de sclérose en plaques (SP). Méthode : Soixante-douze patients, chez qui un diagnostic de SP récurrente rémittante avait été posé, ont été inclus dans l'étude de façon prospective. Le score à l'exploration fonctionnelle du tronc cérébral (BSFS), la partie de l'EDSS qui évalue la symptomatologie du tronc cérébral, a été calculé. L'IRM 1,5T et les séquences T1, T2, PD et FLAIR ont été analysées pour détecter la présence de lésions du tronc cérébral. Les potentiels évoqués auditifs (PÉA) et les potentiels évoqués myogéniques vestibulaires oculaires et cervicaux (PÉMVo et PÉMVc) ont été enregistrés selon un protocole standardisé. Résultats : Parmi les 72 patients inclus dans l'étude, 18 (25\%) avaient des manifestations cliniques d'atteinte du tronc cérébral. L'IRM a mis en évidence une atteinte du tronc cérébral chez 29 patients (40\%). Lors des épreuves neurophysiologiques, 16 patients (22\%) avaient des PÉA pathologiques, 36 patients (50\%) avaient des PÉMVo anormaux, 18 patients (25\%) avaient des PÉMVc anormaux et 45 patients (63\%) avaient des PÉMV (PÉMVo et PÉMVc combinés) anormaux. Les PÉMV ont détecté des lésions du tronc cérébral chez un pourcentage plus élevé de patients que l'examen clinique, l'IRM et les PÉA, ce qui était significatif au point de vue statistique $(<0,0001,0,012$ et $<0,0001$ respectivement). Conclusions : Les résultats de cette étude démontrent que les PÉMV constituent la meilleure méthode de détection des lésions du tronc cérébral dans la SP et qu'ils les détectent significativement mieux que l'examen clinique, les PÉA ou l'IRM.

Can J Neurol Sci. 2014; 41: 346-349

Multiple sclerosis (MS) is a chronic neurological disease, which affects young adults at a time when they have to make most of their personal and professional choices. Predicting the long-term disability of MS is therefore very important for patients as well as neurologists. It has become even more crucial nowadays with more and more treatment options becoming available.

Several prognostic factors of long-term irreversible disability have been described in MS: 1) at onset: ethnicity, sex, age, type of symptoms, and initial course and 2) later on, they include recovery from the initial symptoms, delay to the second neurologic episode, number of relapses in the first few years, and clinical characteristics in the early phase of the disease. ${ }^{1}$ Recent studies have also suggested that brainstem involvement is an important predictor of future disability. ${ }^{2,3}$ These studies have shown that the presence of at least one brainstem lesion increases both the risk of conversion and disability, ${ }^{2}$ and that two or more infratentorial lesions best predicts long-term disability. ${ }^{3}$ Another study has shown, when comparing patients with just the symptomatic brainstem lesion and patients with symptomatic brainstem lesion together with asymptomatic lesions on the magnetic resonance imaging (MRI), the latter had statistically significant higher chance to convert to MS. ${ }^{4}$ Therefore it is very important to detect brainstem lesions early in order to advise patients accordingly.

From the University Hospital Center Zagreb (MKS, IA, VNM, MH), Department of Neurology, Referral Center for Demyelinating Diseases of the Central Nervous System; School of Medicine (MH), University of Zagreb, Zagreb, Croatia. Received October 9, 2013. Final Revisions Submitted November 5, 2013. Correspondence to: Mario Habek, University Department of Neurology, Zagreb School of Medicine and University Hospital Center, Kišpatićeva 12, HR-10000 Zagreb, Croatia.Email: mhabek@mef.hr. 
Table 1: Showing oVEMP, VEMP and MRI are superior to AEP in detection of brainstem lesions

\begin{tabular}{l|l}
\hline AEP versus & P value \\
\hline BSFS & 0.845 \\
\hline oVEMP* & 0.002 \\
\hline cVEMP & 0.839 \\
\hline VEMP* & $<0.0001$ \\
\hline MRI* & 0.026 \\
\hline
\end{tabular}

BSFS brainstem functional system score (part of the EDSS) showing clinical involvement of the brainstem; AEP audiotory evoked potentials, oVEMP ocular vestibular evoked myogenic potentials; cVEMP cervical vestibular evoked myogenic potentials; VEMP vestibular evoked myogenic potentials, MRI magnetic resonance imaging; * indicates statistical significance

The deficiency of the conventional MRI in the detection of brainstem lesions is well known., ${ }^{5,6}$ Vestibular evoked myogenic potentials (VEMP), on the other hand, are recognized as fundamental in the assessment of vestibuloocular and vestibulospinal involvement. Two VEMP tests exist: cervical VEMP (cVEMP) that evaluates integrity of vestibulospinal pathway and ocular VEMPs (oVEMP) that is a manifestation of vestibuloocular reflex. Both oVEMP and cVEMP response parameters demonstrate good test-retest reliability, making them good tools for evaluating brainstem involvement. ${ }^{7}$ Abnormal results of both VEMP in patients with MS implicates lesion of the brainstem, despite normal MRI and/or neurological examination. ${ }^{8,9}$

Therefore we hypothesized that VEMP is superior in detection of brainstem lesions than other conventional measures of brainstem damage: clinical examination, auditory evoked potentials (AEP) and brain MRI.

\section{Patients And Methods}

Patients: Patients with the diagnosis of relapsing-remitting MS according to the 2010 revision of the McDonald's criteria were prospectively included in the study. ${ }^{10}$ Exclusion criteria were ear disease or medications that could influence VEMP results like diazepam or clonazepam. All participants were informed about the details of the experiment and they all signed

Table 3: Showing no difference between cVEMP comparing to other studied parameters

\begin{tabular}{l|l}
\hline cVEMP versus & P value \\
\hline BSFS & 1.000 \\
\hline AEP & 0.839 \\
\hline MRI & 0.054 \\
\hline
\end{tabular}

BSFS brainstem functional system score (part of the EDSS) showing clinical involvement of the brainstem; AEP audiotory evoked potentials, cVEMP cervical vestibular evoked myogenic potentials
Table 2: Showing oVEMP is superior to BSFS and AEP in detection of brainstem lesions

\begin{tabular}{l|l}
\hline oVEMP vesrus & P value \\
\hline BSFS* & 0.003 \\
\hline AEP* & 0.002 \\
\hline MRI & 0.324 \\
\hline
\end{tabular}

BSFS brainstem functional system score (part of the EDSS) showing clinical involvement of the brainstem; AEP audiotory evoked potentials, oVEMP ocular vestibular evoked myogenic potentials; MRI magnetic resonance imaging; $*$ indicates statistical significance

informed consent forms. The Ethical committee of the University Hospital Centre Zagreb approved the study.

All patients were examined by the neurologist with at least five years experience in MS and expanded disability status scale (EDSS) score and brainstem functional system score (BSFS) (part of the EDSS evaluating brainstem symptomatology) were calculated.

MRI: MRI was performed on 1.5T and T1, T2, PD and fluidattenuated inversion recovery (FLAIR) sequences were analyzed for presence of brainstem lesions. A neurologist with at least five years experience in MS reviewed all MRIs.

Auditory evoked potentials: During the experiment participants sat or lay in a relaxed position in a slightly darkened room. Their eyes were closed in order to avoid ocular artifacts. Activity was recorded with four surface disk electrodes. Active electrodes were situated on the mastoids on both sides and referred to the vertex electrode $(\mathrm{Cz})$. Frontal electrode $(\mathrm{Fz})$ was used as ground electrode.

The stimuli were delivered by a pair of headphones. The stimulation rate was $10 \mathrm{~Hz}$ and stimuli were acoustic clicks of $0.1 \mathrm{~ms}$ duration. At the beginning of the experiment the perceptive threshold for each participant for each ear was assessed. During the experiment, the intensity of stimulation delivered to the tested ear was $70 \mathrm{~dB}$ higher than the perceptive threshold. At the same time the white noise with an intensity 30 $\mathrm{dB}$ lower than the intensity delivered to the tested ear was

Table 4: Showing VEMP is superior to BSFS, AEP and MRI in detection of brainstem lesions

\begin{tabular}{l|l}
\hline VEMP versus & P value \\
\hline BSFS* & $<0.0001$ \\
\hline AEP* & $<0.0001$ \\
\hline MRI* & 0.012 \\
\hline
\end{tabular}

BSFS brainstem functional system score (part of the EDSS) showing clinical involvement of the brainstem; AEP audiotory evoked potentials, MRI magnetic resonance imaging; * indicates statistical significance 
delivered to the contralateral ear in order to reduce the effect of the bone conductivity. Each series consisted of 1000 stimuli and was repeated twice for each ear in order to provide reproducibility. Recordings were performed using Medelec Synergy, Oxford Instruments, UK. Automated analysis according to the normative values was performed with the same system.

The results of AEP were regarded either as pathological or normal according to the normative values for the laboratory.

Vestibular evoked myogenic potentials: VEMP was performed as previously described. ${ }^{9}$ The presented stimuli were acoustic clicks of $1 \mathrm{~ms}$ duration at an intensity of $130 \mathrm{~dB}$ sound proof level (SPL) and the stimulation frequency of $1 \mathrm{~Hz}$. Recordings were performed using a Brain Products Brain Vision Recorded and the analysis of the recorded data was performed using a Brain Products Brain Vision Analyzer. We used baseline normalized values of the sternocleidomastoid muscle (SCM) amplitude data instead of the absolute value of amplitude, because absolute amplitude of the evoked response depends on the amplitude of the muscle activity (muscle contraction) and is not reliable measure. The baseline normalized value of amplitude is calculated by dividing the absolute peak to peak amplitude (P13-N23) with mean value of rectified activity of muscle in the period prior the stimulus.

The results of oVEMP and cVEMP were regarded either as pathological or normal according to the normative values for the laboratory.

\section{Statistical analysis}

According to a power analysis for Exact test, where the size of the effect (Effect size) is 0.15 (according to P1 $=0.8$ and $\mathrm{P} 2=$ 0.65 ; information obtained in the pilot study), power of the test $80 \%$ and significance level 0.05 , a total of 72 patients are needed. Analysis was performed using GPower 3.1 program.

Statistic analysis was performed using IBM SPSS 19.0 (Chicago, IL). We used the McNemar's test, which evaluates changes in related or paired binomial attributes. $\mathrm{P}$ values less than 0.05 were considered significant.

\section{RESULTS}

Seventy two patients participated in the study, 48 females and 24 males, aged from 18 to 58 years (median 33 years). Median EDSS was 1.5 (range from 0 to 3.5 ). Median disease duration was 22.5 months (range from 2 to 307 months).

From 72 patients, 18 (25\%) had clinical involvement of the brainstem evident by BSFS equal or greater than 1 . On the other hand, MRI showed brainstem involvement in 29 (40\%) patients. This difference was statistically significant $(\mathrm{p}=0.027)$, indicating that the MRI is superior comparing to clinical examination in evaluating brainstem involvement in MS.

Of the neurophysiological tests, AEP showed pathological results in $16(22 \%)$ patients, oVEMP in $36(50 \%)$ patients, cVEMP in $18(25 \%)$ patients, and VEMP (combination of oVEMP and cVEMP) in $45(63 \%)$ patients. Comparison between each test is presented in Tables 1-4. Vestibular evoked myogenic potentials detected brainstem lesions in a higher percentage than clinical examination, MRI and AEP, which was statistically significant $(<0.0001,0.012$ and $<0.0001$, respectively).

\section{DISCUSSION}

Results of the present study have shown that VEMPs are the optimal method to detect brainstem lesions in MS and that they detect them significantly better than clinical examination, AEP or MRI. Simultaneous oVEMP and cVEMP tests may be a convenient screening tool for assessing crossed vestibulo-ocular reflexes and ipsilateral sacculo-collic reflexes in every MS patient because this is a noninvasive and short diagnostic procedure with very low cost.

Vestibular evoked myogenic potentials are a new method which has only recently been investigated in MS. The percentage of pathological findings, when oVEMP and cVEMP are used in combination, ranges from $50 \%$ to $80 \% 9,11,12$, which is significantly higher percentage than $\mathrm{AEP}^{13}$. This was confirmed by our previous study, which showed that VEMP is superior to AEP in detecting brainstem involvement ${ }^{11}$. These discrepancies between clinical and neurophysiological data (cliniconeurophysiological paradox) and clinical and MRI data (clinicradiological paradox) could be explained by the fact that the information provided by evoked potentials is more related to the function, unlike MRI, which is more related to anatomy. Nevertheless, detection of brainstem lesions is very important in MS, since it has been shown that this is one of the best predictors of conversion from clinically isolated syndrome to MS or for future disability. As all studies showing the importance of brainstem lesions used MRI as a tool to detect brainstem lesions, these results are hard to extrapolate on lesions detected by evoked potentials. ${ }^{2,14}$ In spite of this, combinations of different evoked potentials has shown very high sensitivity and specificity in predicting future disability in MS. ${ }^{15}$

The limitation of this study is that the MRI was done on $1.5 \mathrm{~T}$ MRI, especially knowing that turbo spin-echo, double inversion recovery brain imaging at $3 \mathrm{~T}$ provides the highest overall sensitivity in the detection of infratentorial MS lesions. ${ }^{16}$

Although the present study showed superiority in detection of brainstem lesions by VEMP, long-term capability of VEMP in prediction of future disability remains to be investigated .

\section{REFERENCES}

1. Renoux C. Natural history of multiple sclerosis: long-term prognostic factors. Neurol Clin. 2011;29(2):293-308.

2. Tintore M, Rovira A, Arrambide G, et al. Brainstem lesions in clinically isolated syndromes. Neurology. 2010;75(21):1933-8.

3. Minneboo A, Barkhof F, Polman CH, Uitdehaag BM, Knol DL, Castelijns JA. Infratentorial lesions predict long-term disability in patients with initial findings suggestive of multiple sclerosis. Arch Neurol. 2004;61(2):217-21.

4. Sastre-Garriga J, Tintore M, Rovira A, et al. Conversion to multiple sclerosis after a clinically isolated syndrome of the brainstem: cranial magnetic resonance imaging, cerebrospinal fluid and neurophysiological findings. Mult Scler. 2003;9(1):39-43.

5. Gass A, Filippi M, Grossman RI. The contribution of MRI in the differential diagnosis of posterior fossa damage. J Neurol Sci. 2000;172 Suppl 1:S43-9.

6. Zadro I, Barun B, Habek M, Brinar VV. Isolated cranial nerve palsies in multiple sclerosis. Clin Neurol Neurosurg. 2008;110 (9):886-8

7. Nguyen KD, Welgampola MS, Carey JP. Test-retest reliability and age-related characteristics of the ocular and cervical vestibular evoked myogenic potential tests. Otol Neurotol. 2010;31(5): 793-802. 
8. Eleftheriadou A, Deftereos SN, Zarikas V, et al. The diagnostic value of earlier and later components of Vestibular Evoked Myogenic Potentials (VEMP) in multiple sclerosis. J Vestib Res. 2009;19(1-2):59-66.

9. Gabelic T, Krbot M, Sefer AB, Isgum V, Adamec I, Habek M. Ocular and cervical vestibular evoked myogenic potentials in patients with multiple sclerosis. J Clin Neurophysiol. 2013;30 (1):86-91.

10. Polman CH, Reingold SC, Banwell B, et al. Diagnostic criteria for multiple sclerosis: 2010 revisions to the McDonald criteria. Ann Neurol. 2011;69(2):292-302.

11. Ivankovic A, Nesek Madaric V, Starcevic K, et al. Auditory evoked potentials and vestibular evoked myogenic potentials in evaluation of brainstem lesions in multiple sclerosis. J Neurol Sci. 2013;328(1-2):24-7.

12. Gazioglu S, Boz C. Ocular and cervical vestibular evoked myogenic potentials in multiple sclerosis patients. Clin Neurophysiol. 2012;123(9):1872-9.
13. Japaridze G, Shakarishvili R, Kevanishvili Z. Auditory brainstem, middle-latency, and slow cortical responses in multiple sclerosis. Acta Neurol Scand. 2002;106(1):47-53

14. Sailer M, O'Riordan JI, Thompson AJ, et al. Quantitative MRI in patients with clinically isolated syndromes suggestive of demyelination. Neurology. 1999;52(3):599-606.

15. Invernizzi $\mathrm{P}$, Bertolasi L, Bianchi MR, Turatti M, Gajofatto A, Benedetti MD. Prognostic value of multimodal evoked potentials in multiple sclerosis: the EP score. J Neurol. 2011;258 (11): 1933-9.

16. Wattjes MP, Lutterbey GG, Gieseke J, et al. Double inversion recovery brain imaging at $3 \mathrm{~T}$ : diagnostic value in the detection of multiple sclerosis lesions. AJNR Am J Neuroradiol. 2007; 28(1):54-9. 